\title{
Elevated contextual fear memory by SIRT6 depletion in excitatory neurons of mouse forebrain
}

\author{
Hyopil Kim, Hyun-Seok Kim² and Bong-Kiun Kaang ${ }^{1 *}$
}

\begin{abstract}
A class of NAD-dependent protein deacetylases, the Sirtuin (SIRT) family of proteins is involved in aging, cell survival, and neurodegeneration. Recently, SIRT proteins, including SIRT6, have been reported to be important in learning and memory. However, the role of SIRT6 in excitatory brain neurons in cognitive behaviors is not well characterized. We investigated how cognitive behaviors are affected by genetic SIRT6 depletion in excitatory neurons in the mouse forebrain. We generated a conditional knockout (CKO) mouse line by mating two transgenic lines, Floxed SIRT6 and CaMKIla-Cre. SIRT6 was thus deleted by Cre recombinase in CaMKIla-expressing excitatory neurons. We performed cognitive behavioral tests, focusing on learning and memory, including contextual fear conditioning and Morris-water maze. The freezing level of SIRT6 CKO before the fear conditioning was comparable to that of wild-type littermate controls, while the freezing level after the conditioning was higher in SIRT6 cKO mice. In contrast, the mice showed normal spatial learning and memory in the Morris-water maze. In addition, anxiety and locomotion were also normal in SIRT6 CKO mice. SIRT6 genetic depletion enhanced contextual fear memory without affecting spatial memory. Since a previous report showed that overexpression of SIRT6 reduced contextual fear memory, our results suggest that the expression level of SIRT6 bi-directionally regulates contextual fear memory in mice.
\end{abstract}

Keywords: Sirtuin, SIRT6, Behavior, Learning, Spatial memory, Contextual fear memory

\section{Main text}

Sirtuins (SIRTs) are a class of nicotinamide adenine dinucleotide (NAD)-dependent deacetylases that have been found to be involved in aging and cellular stress in various species [1-3]. There are seven mammalian SIRT proteins with varying localizations and functions. Among the SIRT proteins, SIRT6 is prominently localized in the nucleus and its insufficiency promotes genomic instability and aging $[3,4]$. Furthermore, SIRT6 plays neuroprotective roles, preventing neurodegenerative events [5]. SIRT6 deacetylates histone H3 lysine 9 (H3K9) and this modulates the protective roles [6]. Since epigenetic regulations such as DNA methylation and histone acetylation also mediate learning and memory, SIRT6 would be involved in the processes $[7,8]$.

\footnotetext{
*Correspondence: kaang@snu.ac.kr

${ }^{1}$ Laboratory of Neurobiology, School of Biological Sciences, Seoul National University, Seoul 08826, South Korea

Full list of author information is available at the end of the article
}

Supporting this, SIRT proteins have been implicated in learning and memory. For instance, mice deficient in SIRT1, another SIRT localized in the nucleus as well as the cytoplasm, show deficits in memory and synaptic plasticity such as long-term potentiation [9]. Insufficiency of SIRT3, which is expressed in mitochondria and involved in neuroprotection, results in deteriorated remote memory [10]. The loss of SIRT6 in neuronal progenitors resulted in the accumulation of toxic tau proteins and severe deficits in both associative and non-associative memory [5]. Therefore, SIRT6 is thought to be important for learning and memory. Interestingly, however, overexpression of SIRT6 also impaired longterm contextual fear memory [11].

To further examine the relationship between SIRT6 and learning and memory, we generated a conditional SIRT6 knockout (SIRT6 cKO) by mating Floxed SIRT6 mice with mice expressing Cre recombinase under the control of the $\mathrm{Ca}^{2+} /$ calmodulin-dependent kinase IIa (CaMKIIa) 
promoter. CaMKIIa is a marker of excitatory neurons of the forebrain; hence, CaMKIIa-promoter-driven Cre deletes SIRT6 in those neurons. Mice with a genotype of SIRT6 fl/fl; CaMKIIa-Cre +/- were used as SIRT6 cKO since SIRT6 can be deleted in Cre expressing neurons. Littermate mice with a genotype of SIRT6 fl/fl; CaMKIIa-Cre - /- were used as WT controls (Additional file 1: Figure S1). All the experimental procedures including animal cares are presented in the Additional file 2.

At first, we examined contextual fear memory in SIRT6 cKO mice. We assessed the percentage of the time spent freezing (freezing levels) before and 1 day after shock conditioning. While the freezing levels before shock conditioning were comparable between SIRT cKO and control mice, the post-conditioning freezing level of the cKO mice was higher than that of the WT mice (Fig. 1a, Student's $t$-test and two-way ANOVA; genotype $\mathrm{x}$ shock, $p<0.05)$. There was no significant interaction in the two-way ANOVA test, but the effect of genotype was significant.

In addition, we performed a Morris-water maze test to assess spatial learning and memory. In the training session, the escape latency of cKO mice on day 3 was tended to be lower than that of WT, but the difference was not significant and the latency value was too high, so we trained the mice two more days. The learning curve of the cKO mice during the 5 days of training was comparable to the WT controls, as was their memory in a probe test performed 1 day after the final training day (Recent; Fig. 1b, left and middle, Additional file 3: Figure S2). We performed another probe test 28 days post-training (Remote) because SIRT3 KO mice were reported to have a remote memory deficit. However, remote memory in SIRT6 cKO mice was also comparable to that of WT mice (Fig. 1b, right, Additional file 3: Figure S2).

Finally, we assessed anxiety and locomotive behaviors since the factors can affect various behaviors such as the freezing level. SIRT6 cKO mice showed comparable levels of anxiety in both elevated zero maze and open field tests (Fig. 1c, d). Locomotion in SIRT6 cKO mice also was not significantly different from that in WT mice (Fig. 1d).

In the present study, we assessed the effect of genetic SIRT6 depletion in excitatory neurons on behaviors related to learning and memory. Contextual fear memory was elevated by SIRT6 depletion, contrary to a previous report showing memory impairment following genetic SIRT6 inactivation in neuronal progenitors [5]. However, since the other group targeted total neuronal populations rather than excitatory neurons, the discrepancy may be attributable to SIRT6 depletion in inhibitory neurons resulting in memory impairments. Furthermore, because

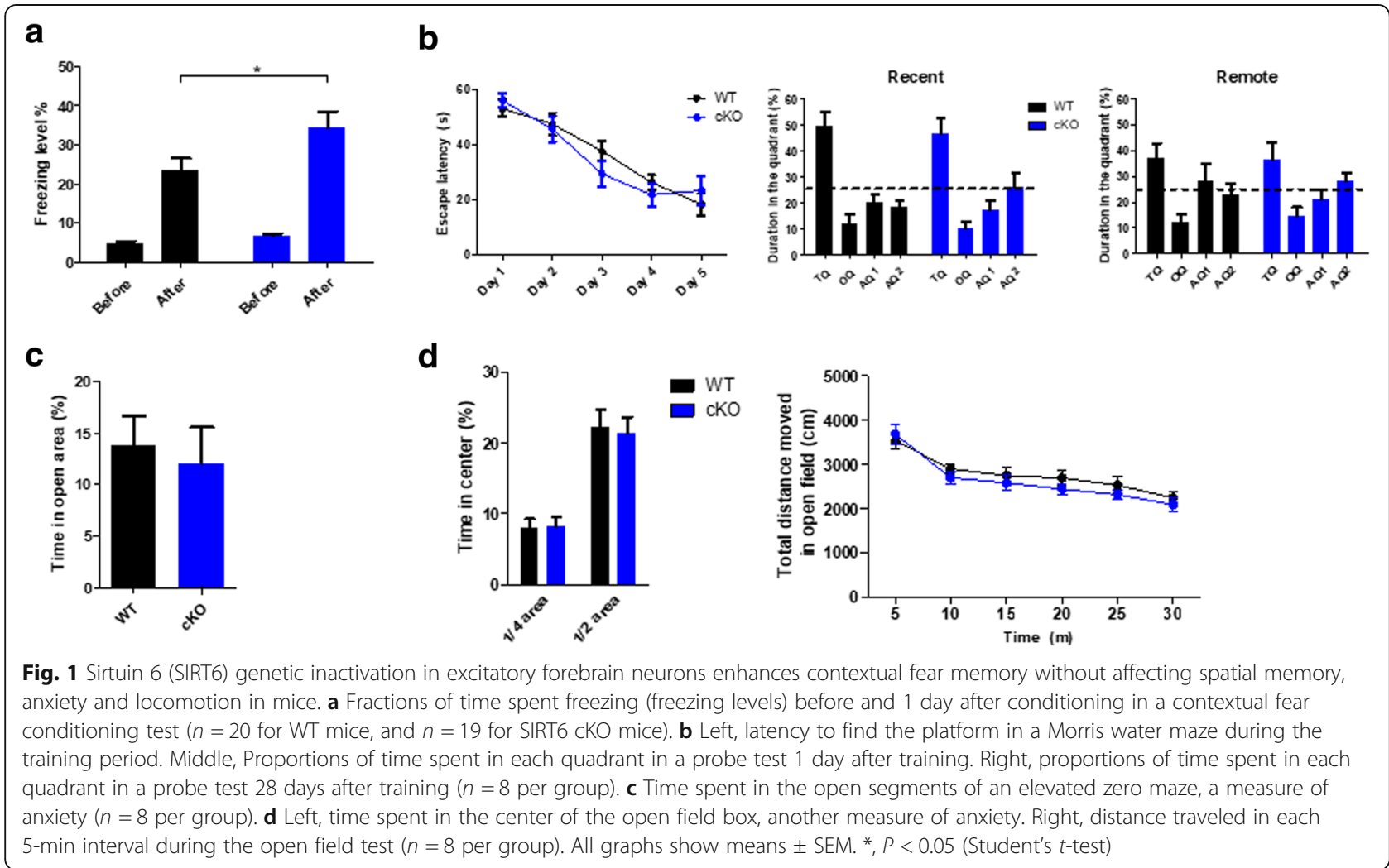


another report [11] showed that SIRT6 overexpression in the CA1 region of the hippocampus impaired contextual fear memory, the memory enhancement observed in our study may indicate specific involvement of SIRT6 function in excitatory CA1 neurons in memory processes in mice. Moreover, because SIRT6 overexpression impaired the Insulin like growth factor (IGF)/Akt signaling pathway, which activates cAMP response element-binding protein (CREB), this pathway may be activated and contribute to the contextual fear memory enhancement in SIRT6 cKO mice $[11,12]$.

Interestingly, unlike contextual fear memory, spatial memory was not affected in SIRT6 cKO mice. Dysregulation of conditioned fear responses are involved in post-traumatic stress disorder (PTSD), hence the selective enhancement of negative memory of SIRT6 cKO mice suggests that reduced SIRT6 activity may be implicated in the disorder. However, in relation with the spatial memory, the possibility of over-training in the Morris-water maze test or another type of spatial memory tests, such as 8 arm maze test can be examined.

\section{Additional files}

Additional file 1: Figure S1. The breeding scheme of $\mathrm{CKO}$ and its littermate controls. (TIF $61 \mathrm{~kb}$ )

Additional file 2: Material and Methods. (DOCX $133 \mathrm{~kb}$ )

Additional file 3: Figure S2. Various measures of spatial memory in the probe tests of the Morris-water maze. (TIF $56 \mathrm{~kb}$ )

\section{Abbreviations}

CaMKIla: Ca2+/calmodulin-dependent kinase Ila; cKO: Conditional knockout; IGF: Insulin-like growth factor; NAD: Nicotinamide adenine dinucleotide; PTSD: Post-traumatic stress disorder; SIRT: Sirtuin

\section{Acknowledgements}

We thank the members of the BKK lab for their technical help.

\section{Funding}

This work was supported by the National Honor Scientist Program of Korea (to B.K.K., NRF2012R1A3A1050385).

\section{Availability of data and materials}

The datasets used and/or analyzed during the current study are available from the corresponding author on reasonable request.

\section{Authors' contributions}

BKK, HSK, and HK conceived and designed the experiments. HSK procured the Floxed-Sirt6 mouse line. HK conducted the behavioral experiments. HK and BKK prepared the manuscript. All authors read and approved the final manuscript.

\section{Ethics approval}

All animal care and experiments, which were conducted according to the policies and regulations for the care and use of laboratory animals, were approved by the Institutional Animal Care and Use Committee of Seoul National University.

\section{Consent for publication}

Not applicable.

\section{Competing interests}

The authors declare that they have no competing interests.

\section{Publisher's Note}

Springer Nature remains neutral with regard to jurisdictional claims in published maps and institutional affiliations.

\section{Author details}

${ }^{1}$ Laboratory of Neurobiology, School of Biological Sciences, Seoul National University, Seoul 08826, South Korea. ${ }^{2}$ The Research Center for Cellular Homeostasis, Ewha Womans University, Seoul 03760, South Korea.

Received: 15 July 2018 Accepted: 16 August 2018

Published online: 06 September 2018

References

1. Ardestani PM, Liang F. Sub-cellular localization, expression and functions of Sirt6 during the cell cycle in HeLa cells. Nucleus. 2012;3:442-51.

2. Burnett C, Valentini S, Cabreiro F, Goss M, Somogyvári M, Piper MD, et al. Absence of effects of Sir2 overexpression on lifespan in C. Elegans and drosophila. Nature. 2011;477:482-5.

3. Gao J, Wang WY, Mao YW, Gräff J, Guan JS, Pan L, Tsai LH, et al. A novel pathway regulates memory and plasticity via SIRT1 and miR-134. Nature. 2010:466(1109):1105.

4. Howitz KT, Bitterman KJ, Cohen HY, Lamming DW, Lavu S, et al. Small molecule activators of sirtuins extend Saccharomyces cerevisiae lifespan. Nature. 2003;425:191-6.

5. Kaluski S, Portillo M, Besnard A, Stein D, Einav M, Zhong L, et al. Neuroprotective functions for the histone deacetylase SIRT6. Cell Rep. 2017; 18:3052-62.

6. Kawahara TL, Michishita E, Adler AS, Damian M, Berber E, Lin M, et al. SIRT6 links histone $\mathrm{H3}$ lysine 9 deacetylation to NF-kappaB-dependent gene expression and organismal life span. Cell. 2009;136:62-74.

7. Yu N, Baek SH, Kaang B. DNA methylation-mediated control of learning and memory. Molecular Brain. 2011:4:5.

8. Kim S, Kaang B. Epigenetic regulation and chromatin remodeling in learning and memory. Exp Mol Med. 2017;49(1):e281.

9. Kim H, Kim S, Choi JE, Han D, Koh SM, Kim HS, et al. Decreased neuron number and synaptic plasticity in SIRT3-knockout mice with poor remote memory. Neurochem Res. 2017:1-7.

10. Mostoslavsky R, Chua KF, Lombard DB, Pang WW, Fischer MR, Gellon L, et al, Genomic instability and aging-like phenotype in the absence of mammalian SIRT6. Cell. 2006;124:315-29.

11. Yin X, Gao Y, Shi HS, Song L, Wang JC, Shao J, et al. Overexpression of SIRT6 in the hippocampal CA1 impairs the formation of long-term contextual fear memory. Sci Rep. 2016;6:18982.

12. Wen-Hua Z, Rémi Q. Insulin-like growth factor-1 (IGF-1) induces the activation/phosphorylation of Akt kinase and CAMP response elementbinding protein (CREB) by activating different signaling pathways in $\mathrm{PC} 12$ cells. BMC Neurosci. 2006;7:51.

Ready to submit your research? Choose BMC and benefit from:

- fast, convenient online submission

- thorough peer review by experienced researchers in your field

- rapid publication on acceptance

- support for research data, including large and complex data types

- gold Open Access which fosters wider collaboration and increased citations

- maximum visibility for your research: over $100 \mathrm{M}$ website views per year

At BMC, research is always in progress.

Learn more biomedcentral.com/submission 